\title{
One-pot Synthesis of Cellulose / Silver Nanoparticle Fibers and their Antibacterial Application
}

\author{
Zhinong Zeng, Shujuan Yang, Lei Zhang, Rong Tang, and Liping Zhang* \\ A simple, novel method was developed for synthesizing cellulose (CE) \\ fibers doped with silver nanoparticles (Ag NPs) in the green solvent \\ tetrabutylammonium hydroxide / dimethyl sulfoxide / $\mathrm{H}_{2} \mathrm{O}$ at room \\ temperature. Tetrabutylammonium hydroxide accelerated the reduction of \\ $\mathrm{Ag}^{+}$to $\mathrm{Ag}$ by the cellulose chains, yielding $\mathrm{Ag}$ NPs in cellulose solution \\ stabilized using polyethyleneimine (PEI). After $24 \mathrm{~h}$, almost all the $\mathrm{Ag}^{+}$was \\ reduced to Ag NPs. The influences of silver nitrate concentration, reaction \\ time, and stabilizer on the formation of Ag NPs were investigated by UV- \\ vis spectrophotometry. The prepared smooth and dense cellulose / Ag NP \\ fibers showed high mechanical properties, with a tensile strength of 304.3 \\ $\mathrm{MPa}$ and an elongation at break of $22.1 \%$. The fibers exhibited excellent \\ antibacterial activities against Escherichia coli and Staphylococcus \\ aureus, with more than $99 \%$ of $E$. coli bacteria killed by Ag NP / cellulose \\ fibers. The synthesis procedure offers a general and mild approach to \\ designing materials of almost any shape.
}

Keywords: Cellulose solution; Silver nanoparticle; Tetrabutylammonium hydroxide; Polyethyleneimine; Antibacterial properties

Contact information: Department of Chemistry and Chemical Engineering, MOE Engineering Research Center of Forestry Biomass Materials and Bioenergy, Beijing Forestry University, Beijing 100083, China;

*Corresponding author: zhanglp418@163.com

\section{INTRODUCTION}

Cellulose - one of the most common organic polymers - is considered an almost inexhaustible raw material having the potential to meet the demand for environmentally friendly biocompatible materials. Cellulose products are widely used in medical supplies, textiles, and packaging products (Rogina-Car et al. 2014; Li and Huang 2016). However, cellulose products that are hygroscopic and porous provide substrates on which bacteria can breed, which can lead to disease (Kontturi et al. 2013). It is therefore essential and of great interest to prepare cellulose products with excellent antibacterial properties (Tsai et al. 2017). Silver nanoparticles (Ag NPs) have been the subject of intensive research in recent years. Due to their exceptional properties, including large surface area and high antibacterial activity (Liang et al. 2014; Felix et al. 2016), Ag NPs have been applied in a diverse range of fields including biomedicine, optical electronics, food packing, and textiles (Chaloupka et al. 2010; Echegoyen and Nerín 2013; Zhang et al. 2015). Silver nanoparticles effectively eliminate bacteria at relatively low concentrations that are not toxic to human cells. Additionally, to the best of the authors' knowledge, bacterial resistance to Ag NPs has not been reported to date (Yuan et al. 2012). Introducing silver nanoparticles into cellulose matrices to provide them with antibacterial function has therefore been a common approach (Tsai et al. 2017).

Recently, there have been numerous reports of cellulose being modified with $\mathrm{Ag}$ NPs using different methods. For example, there has been increasing research interest in 
the preparation of cellulose / Ag NPs composites used in dipping methods. The experimental process requires a large amount of $\mathrm{Ag}$ to immerse the film, which would cause a mass of silver waste (Wu et al. 2014). In addition, the binding force between $\mathrm{Ag}$ NPs and cellulose is weak, such that the Ag NPs absorbed on the fibers surface can easily fall off (Taurozzi et al. 2008; Nangmenyi et al. 2009). Although adding Ag NPs directly to the cellulose solution is an economical and robust method without silver waste, evenly dispersing dry Ag NP powder in cellulose solution is a substantial challenge (RacRumijowska et al. 2017). To address this problem, Fakirov proposed the concept of conversion instead of addition, in which well-dispersed nanoparticles are produced directly on the matrix (Kannan et al. 2016). Therefore, in this study, Ag NPs were directly produced by in situ synthesis in cellulose solution under protection of polyethyleneimine (PEI). The PEI can stabilize the nanoparticle size and shape and prevent potential nanoparticle aggregation (Lee et al. 2011). Rac-Rumijowska et al. (2017) prepared nano silver particles in NMMO cellulose solution and PEI as stabilizer better than poly(vinylpyrrolidone) (PVP) or polyethylene glycol (PEG). In addition, methods for the preparation of Ag NPs based on the reduction of $\mathrm{Ag}$ ions using toxic or dangerous reducing agents have been reported and often also require harsh reaction conditions such as high temperature and pressure (Seo et al. 2013; Zhang et al. 2015). For example, Tran et al. (2016) prepared a cellulose and keratin regenerated film using 1-butyl-3-methylimidazolium chloride ([BMIM]Cl) as the solvent and $\mathrm{NaBH}_{4}$ as the reducing agent, at a temperature greater than $110{ }^{\circ} \mathrm{C}$. In the experiment, sliver nanoparticles were synthesized at room temperature with no additional reducing agent: The cellulose was both the matrix and the reducing agent, and the reaction conditions were mild.

Unlike many other polymers, cellulose is difficult to dissolve in general solvents; however, most of its applications rely on an effective dissolution process (Miao et al. 2016). Quaternary ammonium salt ionic liquids, such as tetrabutylammonium hydroxide (TBAH) and tetrabutylammonium acetate (TBAA), have attracted increasing attention in recent years. Tetrabutylammonium hydroxide is easily recycled and does not lead to the degradation of the polysaccharide (Medronho et al. 2019). Compared with other solvents, such as aqueous $\mathrm{NaOH}$ /urea or nonaqueous ionic liquid, $\mathrm{TBAH}$ is an efficient, green, cheap, and mild solvent that can dissolve cellulose quickly at room temperature (Chen et al. 2018). Additionally, dimethyl sulfoxide (DMSO) can be added as a co-solvent to improve the ability of TBAH to dissolve solutes. Cao et al. (2018) obtained a cellulose solution with a concentration of $4.8 \mathrm{wt} \%$ using TBAH/DMSO$/ \mathrm{H}_{2} \mathrm{O}$ and then regenerated cellulose films at room temperature. In the present experiment, different ratios of solvent were used to achieve higher-concentration cellulose solutions to obtain regenerated cellulose fibers. To date, there have been no reports of the antibacterial modification of cellulose using TBAH as a solvent.

Herein, a facile, one-pot, green method of synthesizing Ag NPs using cellulosewhich was dissolved in TBAH solvent - as both the reducing agent and matrix is reported for the first time. When the cellulose pulp was dissolved, the hydrogen-bonding network was destroyed, and many reducing ends were exposed, which enhanced the reduction capability. Additionally, alkali from the dissolving system markedly accelerated the reduction of $\mathrm{Ag}^{+}$by cellulose at room temperature. Both factors are thought to contribute to the accelerated reduction of $\mathrm{Ag}^{+}$to $\mathrm{Ag}$. Therefore, ionic liquids not only act as solvents but also act as accelerators in the reduction of silver ions. Polyethyleneimine was added due to Ag NPs having high specific surface area and high yield. The mixture allowed the preparation of regenerated fibers using a dry-wet spinning process at room temperature.

Zeng et al. (2021). "Cellulose / Ag nanoparticle fibers," BioResources 16(2), 3360-3376. 3361 
The influences of silver nitrate concentration, reaction time, and stabilizer on the formation of the Ag NPs were investigated by UV-vis spectrophotometry. The viscosity of the mixed solution was suitable for spinning and prepared excellent fibers. X-ray diffraction (XRD) and Fourier-transform infrared (FTIR) analysis were used to determine the effects of the Ag NPs on the fibers. The antibacterial activities of the Ag NP / cellulose fibers against Escherichia coli and Staphylococcus aureus were demonstrated using the inhibition zone method and the shake flask test method, and the fibers were found to have excellent antibacterial activity against $S$. aureus and E. coli.

\section{EXPERIMENTAL}

\section{Materials}

Hardwood pulpboard (with a degree of polymerization of 750) was purchased from Senbo (Shandong, China). Silver nitrate was purchased from Sinopharm Chemical Reagent Co., Ltd. (Shanghai, China). Tetrabutylammonium hydroxide (55 wt $\%$ in water) and tetrabutylammonium acetate were purchased from Tokyo Chemical Industry (Tokyo, Japan). Polyethyleneimine with an average molecular weight of $10 \mathrm{kDa}$ and DMSO were purchased from Shanghai Macklin Biochemical Co., Ltd (Shanghai, China). All chemicals used were analytical grade and did not undergo further purification. Escherichia coli (8099) and Staphylococcus aureus (ATCC6538) were purchased from the China Center for Type Culture Collection (CCTCC) (Wuhan, China).

\section{Preparation of Ag NP / cellulose solution}

The cellulose (CE) was dried at $80{ }^{\circ} \mathrm{C}$ for $12 \mathrm{~h}$ to remove moisture. Tetrabutylammonium hydroxide (55 wt\%) and DMSO were mixed in a 3:7 weight ratio to yield the solvent. Cellulose was then dissolved in $30 \mathrm{~mL}$ of the ionic liquid solvent mixture at $30{ }^{\circ} \mathrm{C}$ to yield a 9.5 -wt $\%$ solution and stirred vigorously for $3 \mathrm{~h}$ to obtain a transparent solution. Set amounts of $\mathrm{AgNO}_{3}$ and PEI (weight ratio $\mathrm{AgNO}_{3} / \mathrm{PEI}=1: 5$ ) were added to the cellulose solution (the weights of $\mathrm{AgNO}_{3}$ were $0.015 \mathrm{wt} \%, 0.03 \mathrm{wt} \%$, and $0.06 \mathrm{wt} \%$, respectively, according to the quality of cellulose the solution), First, the PEI was added to the cellulose solution. Then, after thorough stirring for $0.5 \mathrm{~h}$, ionic silver was added. The reaction was continued for $2 \mathrm{~h}$ to uniformly disperse the $\mathrm{AgNO}_{3}$ and PEI in the cellulose solution. The prepared mixed solution was then sealed for $24 \mathrm{~h}$ at room temperature to yield the spinning solution. An Ag NP / cellulose solution without added PEI was also prepared.

\section{Preparation of Ag NP / cellulose fibers}

The obtained Ag NP / cellulose solution was centrifuged at $4000 \mathrm{rpm}$ for $10 \mathrm{~min}$ to remove the impurities and bubbles. The Ag NP / cellulose blend fibers were spun through a laboratory scale dry-jet wet spinning apparatus. The air gap distance was $25 \mathrm{~mm}$, the spinning speed was $0.3 \mathrm{~mL} / \mathrm{m}$, and the drawing ratio for the blend fibers was 1.7 . The cellulose / Ag NP solution was spun into a coagulation water bath at a temperature of 25 ${ }^{\circ} \mathrm{C}$, and then the blend fibers were stretched in the coagulation bath (Zhong et al. 2017). The synthetic fibers were soaked in deionized water for $48 \mathrm{~h}$, and the water was changed every $8 \mathrm{~h}$ to remove the residual reagents, after which the Ag NP / cellulose blend fibers were obtained (R1, R2, and R3). The pure regenerated cellulose fiber was denoted R0. The fibers were coded as $\mathrm{R} 1, \mathrm{R} 2$, and $\mathrm{R} 3$, when the $\mathrm{AgNO}_{3}$ concentrations of the mixture 
solution were $0.015 \mathrm{wt} \%, 0.03 \mathrm{wt} \%$, and $0.06 \mathrm{wt} \%$, respectively (Table 1 ).

Table 1. Compositions of Spinning Solutions

\begin{tabular}{|c|c|c|c|c|}
\hline $\begin{array}{c}\text { Fiber } \\
\text { Sample }\end{array}$ & $\begin{array}{c}\text { Solution } \\
\text { Sample }\end{array}$ & $\begin{array}{c}\text { Cellulose } \\
(\text { wt\%) }\end{array}$ & $\begin{array}{c}\mathrm{AgNO}_{3} \\
(\mathrm{wt} \%)\end{array}$ & $\begin{array}{c}\mathrm{AgNO}_{3} / \mathrm{PEI} \\
\text { Blend Ratio }\end{array}$ \\
\hline $\mathrm{R} 0$ & $\mathrm{R} 0^{\prime}$ & 9.5 & 0 & 0 \\
\hline $\mathrm{R} 1$ & $\mathrm{R} 1^{\prime}$ & 9.5 & 0.015 & $1: 5$ \\
\hline $\mathrm{R} 2$ & $\mathrm{R} 2^{\prime}$ & 9.5 & 0.03 & $1: 5$ \\
\hline $\mathrm{R} 3$ & $\mathrm{R} 3^{\prime}$ & 9.5 & 0.06 & $1: 5$ \\
\hline
\end{tabular}

wt\% according to the weight of aqueous solution

\section{Antimicrobial Activity Studies}

The antimicrobial activities of the composite fibers were investigated using the inhibition zone method with $E$. coli and $S$. aureus. The bacterial solution, which achieved an exponential growth phase, was diluted to a cell concentration of $10^{5} \mathrm{CFU} / \mathrm{mL}$. The fibers were cut into bar shapes and formed into a circular sheet with a diameter of $12 \mathrm{~mm}$ using a specific tableting mold. Diluted bacterial solution $(1.0 \mathrm{~mL})$ was then inoculated onto the plates, and samples were pressed into close contact with agar culture medium and cultivated at $37^{\circ} \mathrm{C}$ for $24 \mathrm{~h}$. The zones of bacterial inhibition were then monitored. The antibacterial performances of the different fibers were evaluated through the diameters of the inhibitory zones (Yang et al. 2020).

In the shake flask test method, a 0.1-g test specimen that was cut into pieces was added to a test flask containing $10 \mathrm{~mL}$ of the bacterial inoculum after sterilizing by autoclaving for $20 \mathrm{~min}$ at $121^{\circ} \mathrm{C}$. The suspension of bacteria cells had a final density of $10^{4} \mathrm{CFU} / \mathrm{mL}$. Then, all of the sealed flasks were shaken at $120 \mathrm{rpm}$ and $37^{\circ} \mathrm{C}$ for $24 \mathrm{~h}$. The bacterial solutions were subsequently diluted and spread onto LB agar plates. The plates were cultivated at $37{ }^{\circ} \mathrm{C}$ for $24 \mathrm{~h}$ (Yang et al. 2020). The percentage reduction of microorganisms after $24 \mathrm{~h}$ of incubation was calculated using Eq. 1,

$$
Y=\left(W_{\mathrm{t}}-Q_{\mathrm{t}}\right) / W_{\mathrm{t}}
$$

where $Y$ is the antibacterial rate, $W_{\mathrm{t}}$ is the number of microorganisms $(\mathrm{CFU} / \mathrm{mL})$ in the control sample after $24 \mathrm{~h}$ of incubation, and $Q_{\mathrm{t}}$ is the number of microorganisms $(\mathrm{CFU} / \mathrm{mL})$ in the tested sample after $24 \mathrm{~h}$ of incubation.

\section{Characterization}

The rheological properties of the spinning solution were characterized using a rotary rheometer (CVO-100, Bohlin, Malvern, UK). The prepared spinning solution was poured onto the rheometer platform, and the static rheological properties of the spinning solution were tested with a cone clamp with a diameter of $40 \mathrm{~mm}$ and a cone angle of $2^{\circ}$. The shear rate was $0.1 \mathrm{~s}^{-1}$ to $100 \mathrm{~s}^{-1}$.

The Ag NP solution was diluted to an appropriate concentration and then poured into a colorimetric dish for testing. The formation of Ag NPs was confirmed using a UVvis spectrophotometer (PerkinElmer Lambda 650, Waltham, MA, USA) with a scanning wavelength range from $300 \mathrm{~nm}$ to $800 \mathrm{~nm}$ using a 1-cm optical path at ambient temperature.

The Ag NP / cellulose blend fiber (R3), which was carefully milled with a mortar, was added to an appropriate amount of ethanol. After sonicating for 30 minutes, a drop of the ethanol solution was dropped onto a 230-mesh copper mesh. Transmission electron microscopy (TEM) images were acquired using a JEM-1010 transmission electron 
microscope (JEOL, Tokyo, Japan) at an accelerating voltage of $300 \mathrm{kV}$. The size of the particles was measured by Nano Measure software (Visual Basic 6.0, Shanghai, China).

The presence of functional groups in the Ag NP / cellulose blend fibers was investigated using an FTIR spectrometer (Bruker Vector 33) in the $4000 \mathrm{~cm}^{-1}$ to $500 \mathrm{~cm}^{-1}$ range at a resolution of $4 \mathrm{~cm}^{-1}$ using the $\mathrm{KBr}$ method.

To study the effect of Ag NPs on the crystallinity of the nanocomposite fibers, XRD measurements were performed using an X-ray diffractometer (Bruker D8 Advance, Brook, Germany) at $40 \mathrm{kV}$ and $40 \mathrm{~mA}$ with $\mathrm{Cu}-\mathrm{K} \alpha$ radiation. The scan range was from $6^{\circ}$ to $80^{\circ}$, at a scanning rate of $2 \% \mathrm{~min}$. The crystallinity index of the matrix in the nanohybrid fibers was calculated using the peak height method (Eq. 2),

$$
\text { Crystallinity index }(\%)=\left(I_{020}-I_{\mathrm{am}}\right) / I_{020}
$$

where $I_{020}\left(2 \theta=21.6^{\circ}\right.$ for cellulose II $)$ is the intensity of the crystalline fraction, and $I_{a m}$ is the intensity of the amorphous region $\left(2 \theta=16^{\circ}\right.$ for cellulose II) (Fu et al. 2017).

The cross sections and surfaces of the fibers were characterized by scanning electron microscopy (SEM, S-3000N, Hitachi, Tokyo, Japan). The fibers were frozen in liquid nitrogen and immediately snapped. The best sample was chosen, and a single fiber was covered with a platinum layer. Elemental and distribution maps of the Ag NPs in the cross sections of the fibers were obtained using energy dispersive spectroscopy (EDS) from SEM. (Model, Manufacturer, City, Country).

The mechanical properties of the fibers were tested using an electronic tensile tester (Instron 5848, Norwood, MA, USA). The stretching rate was $10 \mathrm{~mm} / \mathrm{min}$, with a $10-\mathrm{mm}$ gauge length. The diameter of the fiber was measured using a microscope (XPV-25, Pudan, Shanghai, China). The tensile strength and elongation were calculated as the averages of at least 20 measurements from stress-strain curves.

\section{RESULTS AND DISCUSSION}

\section{Effect of Factors on the Formation of Ag NPs}

Effect of $\mathrm{AgNO}_{3}$ content on the formation of $\mathrm{Ag} \mathrm{NPS}$

The UV-vis spectra of the cellulose silver nanoparticles solution are shown in Fig. 1. As shown in Fig. 1a, the typical characteristic peak of Ag NPs appeared at approximately $417 \mathrm{~nm}$, which confirmed the presence of the Ag NPs in the cellulose solution kept at room temperature for $24 \mathrm{~h}$. Because of the surface plasmon resonance absorption characteristics of precious metals, Ag NPs have unique absorption bands at $390 \mathrm{~nm}$ to $440 \mathrm{~nm}$ (Song and Kim 2009; Johnston and Nilsson 2012). With the increase of $\mathrm{AgNO}_{3}$ concentration (0.015 $\mathrm{wt} \%$ to $0.06 \mathrm{wt} \%$ ), the intensity of the absorption peak gradually increased, which indicated that an increasing number of Ag NPs were formed in the system, as the intensity of the absorption peak depends on the concentration of Ag NPs.

\section{Change of Ag NPs in solution with time and the effect of stabilizer}

The influences of time and stabilizer on the Ag NPs are shown in Fig. 1b. When mixing had just ended, there was a characteristic absorption peak in the UV-vis spectrum, suggesting that few Ag NPs had been generated at that point. The peak intensity increased with reaction time. After $24 \mathrm{~h}$ of storage at room temperature, a higher absorption peak appeared, which indicated that more Ag NPs were produced as a result of reduction by cellulose at room temperature. However, due to the high concentration of Ag NPs - which 
have a large surface area-generated in the cellulose solution, after $48 \mathrm{~h}$, the absorption peak intensity decreased substantially.
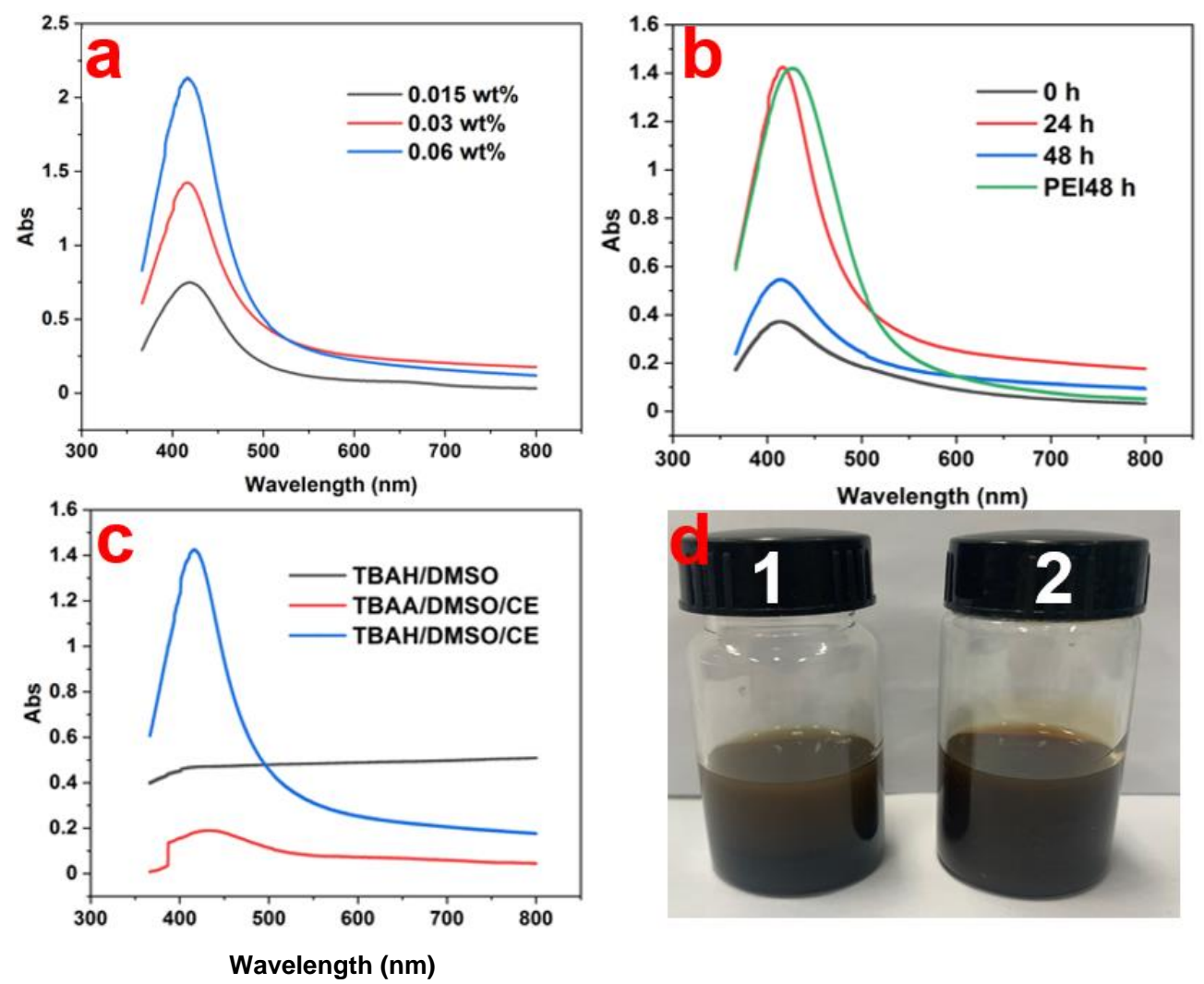

Fig. 1. (a) UV-vis spectra of Ag NPs prepared from solutions with different $\mathrm{AgNO}_{3}$ concentrations: $0.015 \mathrm{wt} \%, 0.03 \mathrm{wt} \%$, and $0.06 \mathrm{wt} \%$; (b) UV-vis spectra of Ag NPs at different times $(0.03 \mathrm{wt} \%$ $\mathrm{AgNO}_{3}$ solution); (c) UV-vis spectra of Ag NPs prepared under different conditions; and (d) digital camera photo: (1) Ag NP / cellulose solution without added PEl after $48 \mathrm{~h}$ and (2) Ag NP / cellulose solution with added PEI after $48 \mathrm{~h}$

Figure 1d (1) shows that the cellulose / Ag NP solution was stratified, with larger Ag NPs deposited on the bottom. Thus, the generation of Ag NPs is mainly divided into two stages: Ag NPs are first generated $(0 \mathrm{~h}$ to $24 \mathrm{~h})$ and then they agglomerate as the generated Ag NPs' concentration increases ( $24 \mathrm{~h}$ to $48 \mathrm{~h}$ ). To prevent the aggregation of Ag NPs, PEI was added to stabilize the cellulose solution. As a result, the absorption peak intensity of the Ag NPs did not change at $48 \mathrm{~h}$ compared to $24 \mathrm{~h}$, and the cellulose solution did not exhibit stratification, as shown in Fig. 1d (2). These findings confirmed that PEI was an effective stabilizer for the reaction.

Roles of cellulose and TBAH in the synthesis of Ag NPs

To investigate the roles of cellulose and TBAH in the synthesis of the Ag NPs, contrast experiments were conducted. When $\mathrm{Ag}^{+}$was added to the TBAH/DMSO/ $\mathrm{H}_{2} \mathrm{O}$ solution (at the same concentration) in the absence of cellulose, no Ag NPs were obtained, as indicated by the UV-vis spectra (Fig. 1c). Additionally, the characteristic absorption band of Ag NPs had a much lower intensity when the solvent was substituted by TBAA/DMSO (Fig. 1c) (the reaction temperature was $55{ }^{\circ} \mathrm{C}$ due to the dissolution characteristics of TBAA, while all other reaction conditions were the same). When DMSO 
is used as a co-solvent, TBAH and TBAA are highly efficient cellulose solvents. These results suggest that cellulose played a crucial role in the synthesis of the Ag NPs, as the TBAH greatly increased the reducing power of the cellulose. The above analysis shows that PEI was used as a stabilizer to prevent the aggregation of Ag NPs, and the cellulose chains acted as the reducing agent in the TBAH solvent.

\section{Formation Mechanism of Ag NPs}

Based on the described results, a possible formation mechanism for cellulose / Ag NP fibers using TBAH solvent as an accelerant is proposed in Fig. 2. Cellulose is a natural polysaccharide containing many hydroxyl groups, which can form intramolecular and intermolecular hydrogen bonds, thus limiting its reducing ability. However, when the cellulose pulp was dissolved, the hydrogen bond network was destroyed, and many reducing ends were exposed, resulting in better reducing ability (Dong et al. 2015).

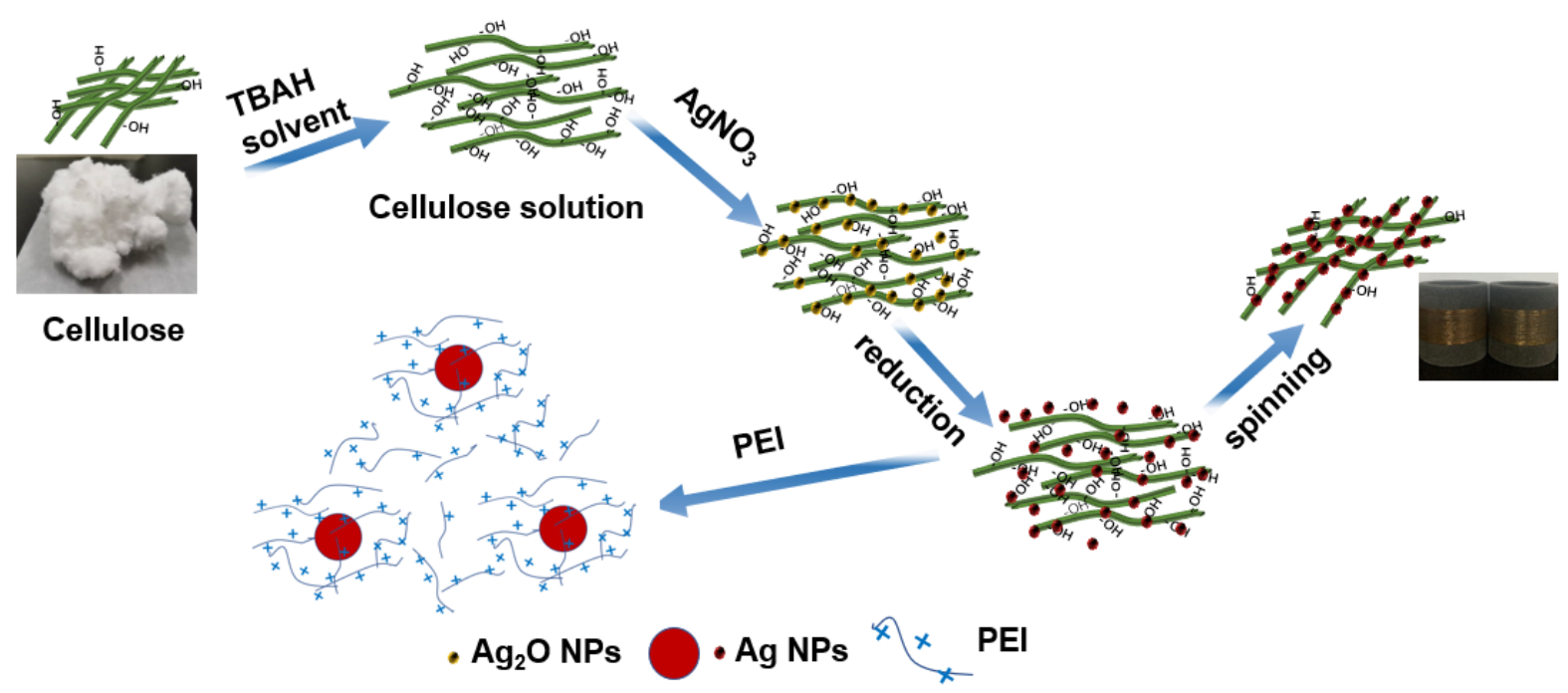

Fig. 2. Formation mechanism of $\mathrm{Ag} \mathrm{NP} /$ cellulose fibers using $\mathrm{TBAH} / \mathrm{DMSO} / \mathrm{H}_{2} \mathrm{O}$ solvent

Meanwhile, when the $\mathrm{AgNO}_{3}$ was added to the cellulose solution, $\mathrm{Ag}_{2} \mathrm{O}$ particles were formed rapidly, as $\mathrm{Ag}^{+}$ions tend to form insoluble $\mathrm{Ag}_{2} \mathrm{O}$ particles in alkaline conditions. Importantly, the reduction of $\mathrm{Ag}_{2} \mathrm{O}$ to $\mathrm{Ag}$ is much easier than the reduction of $\mathrm{Ag}^{+}$to $\mathrm{Ag}$, as widely reported in the literature (Nishimura et al. 2011). Under the synergy of the above two aspects, $\mathrm{Ag}^{+}$ions were reduced by cellulose at room temperature. The PEI molecules, which contain many amino groups in the long molecular chain, act as stabilizer in the course of the metal nanoparticle synthesis (Although PEI has a certain degree of reduction, the synthesis of Ag NPs with PEI is not practical at room temperature (Lee et al. 2011).) This behavior created a simple reaction pathway for the facile formation of the Ag NPs / cellulose.

\section{Basic Characterization Analysis}

The spinning solution's viscosity has a substantial influence on the spinning process and the resultant fiber morphology. Figure 3a shows the change of spinning solution viscosity with shear rate for solutions with different Ag NP contents. The cellulose solutions containing different Ag NP concentrations all exhibited shear thinning, which is a notable feature of polymers (Liu et al. 2011). The findings showed that the properties of 
the cellulose solution after the Ag NPs were generated were similar to those of a typical polymer solution, which, to a certain extent, implied that the cellulose solution was still stable after adding silver nitrate. Figure 3 a also suggests that the viscosity of the cellulose solution increased with the increasing concentration of Ag NPs generated in solution, as the Ag NPs may participate in the physical entanglement of the cellulose in the solution.
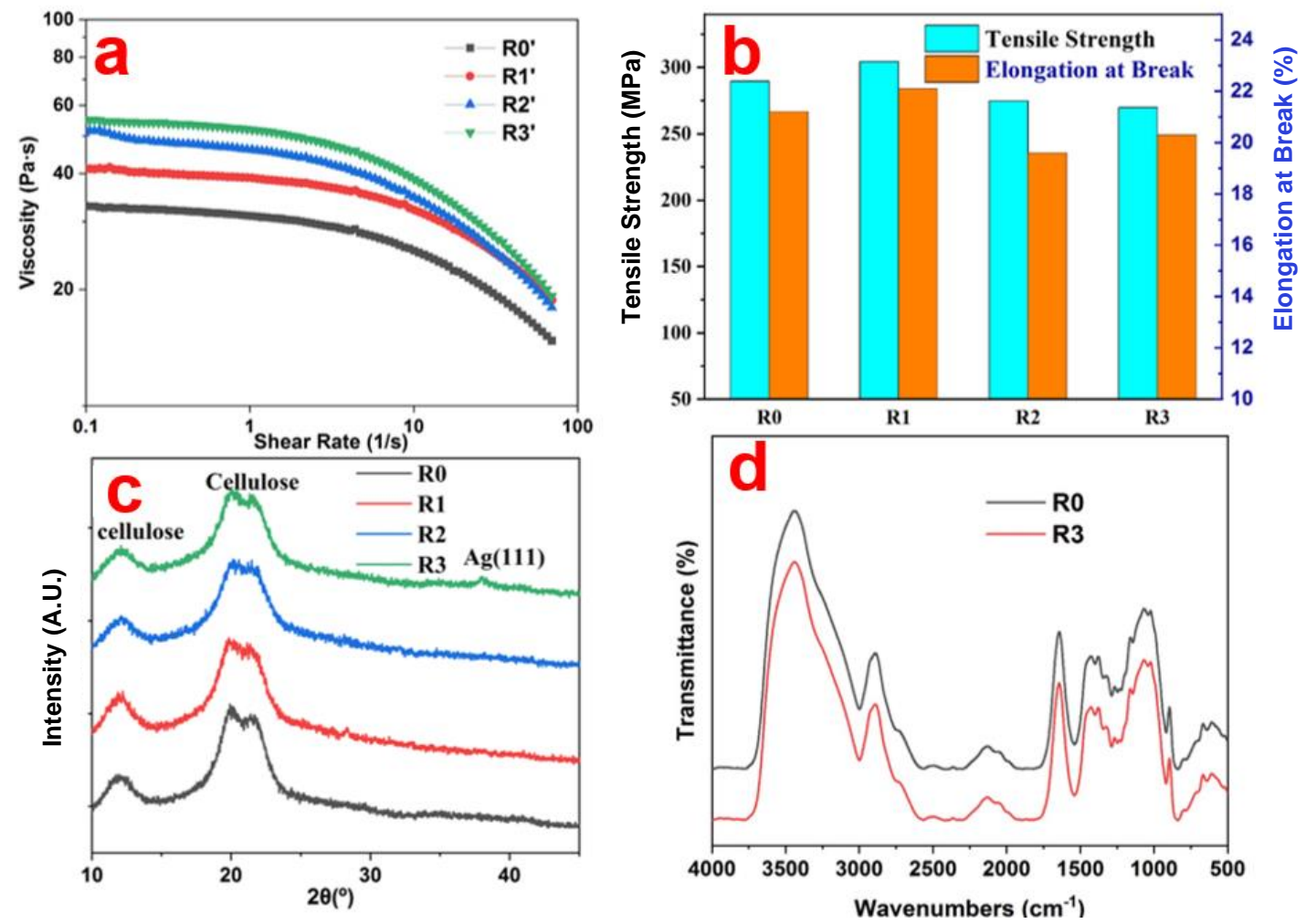

Fig. 3. (a) Viscosities of R0' to R3' solutions for prepared R0 to R3 fibers, (b) tensile properties of composite fibers, (c) XRD patterns of pure cellulose and composite fibers with different silver contents, and (d) FTIR spectra for pure regenerated cellulose fiber and composite fiber (R3)

Mechanical properties constitute an important aspect of cellulose / Ag NP composites due to their direct relation to applicability. The mechanical properties of the composite fibers with different Ag NP contents were measured at room temperature. The results for tensile strength and elongation at break are summarized in Fig. 3b. Pure regenerated cellulose fibers had a tensile strength of $289.5 \mathrm{MPa}$ and an elongation at break of $21.3 \%$, while the mechanical properties of the R1 fibers were slightly greater, at 304.3 $\mathrm{MPa}$ and $22.1 \%$, respectively. The good mechanical properties of the R1 fibers can be explained by the uniform dispersion of the Ag NPs in the cellulose and the good interfacial interaction between them (Chen et al. 2020). The mechanical properties of the R2 and R3 fibers were slightly lower. In general, the prepared regenerated cellulose fibers had good mechanical properties that met the requirements of industrial applications.

To examine the effects of Ag NPs on the crystallinity of the matrix and confirm the formation of Ag NPs in the fibers, X-ray diffractograms of the matrix and nanocomposite fibers prepared using different-concentration $\mathrm{AgNO}_{3}$ source solutions were acquired and are shown in Fig. 3c. All four fibers had three common intensity peaks at $2 \theta=12^{\circ}, 20^{\circ}$, and $21.6^{\circ}$, corresponding to the $(1 \overline{1} 0),(110)$, and $(020)$ planes of cellulose II, respectively, 
which is similar to aqueous $\mathrm{NaOH} /$ urea systems (French 2014).

The addition of silver ions did not induce changes in the crystal structure of cellulose. Additionally, the R3 composite showed a diffraction peak at $2 \theta=38.1^{\circ}$, which was assigned to the (111) peaks, representing the structure of Ag according to the standard card (JCPDS Card No. 4-783). This result indicated that Ag metal was successfully synthesized in the cellulose / Ag NP fibers. The results matched well with the UV-vis result and further demonstrated the successful preparation of the Ag NP / cellulose composite fibers. The peak attributed to cubic silver was not clearly observed in the patterns for the $\mathrm{R} 1$ and R2 fibers, which could be due to the low Ag content. Calculated from the diffractograms - the degree of crystallinity of the cellulose fibers was $57.1 \%$, and the crystallinities of the cellulose / Ag NP fibers were 52.81\%, 52.46\%, and $51.55 \%$, respectively.

The intensities of the diffraction peaks for R1, R2, and R3 were slightly lower than that of the matrix alone. This result was because the crystal structure of cellulose was partially destroyed in the oxidation process. Alternatively, it may indicate that the Ag NPs hindering crosslinking during cellulose regeneration, which affects its crystallinity. Thus, the crystal structure and degree of crystallinity of cellulose were not altered obviously during the in-situ synthesis of the Ag NPs.

Fourier-transform infrared spectra were measured to investigate the effects of the Ag NPs on the structure of cellulose and are presented in Fig. 3d. The spectra of the matrix and the nanocomposite made using $0.06 \mathrm{wt} \%$ aqueous $\mathrm{AgNO}_{3}$ solution are separated for clarity. Peaks were characteristic of cellulose and were visible in the spectra. The peak at $3335 \mathrm{~cm}^{-1}$ was attributed to the $\mathrm{OH}$ group, and the peaks at $2900 \mathrm{~cm}^{-1}, 1420 \mathrm{~cm}^{-1}$, and 1365 $\mathrm{cm}^{-1}$ correspond to $\mathrm{C}-\mathrm{H}$ bonds. The cellulose / Ag NP fibers did not exhibit any additional peaks compared with the pure fibers. This result confirmed that the silver in the fibers was in its metallic form and not in the form of potential metal-organic compounds. The corresponding peak of PEI was not present, which indicates that PEI was removed from the regenerated fiber to a certain extent.

\section{Morphology of Ag NP / Cellulose Fibers and Ag NPs}

The morphologies of the Ag NP / cellulose nanocomposites were characterized by SEM, as shown in Fig. 4. The SEM images showed that all fibers had smooth surfaces and dense cross sections that showed no micropores, even after modification. This maintenance of fiber smoothness was possible as the whole fiber was doped, and the uniformity of the solution was well maintained during the spinning process. The diameter of the fibers was approximately $60 \mu \mathrm{m}$, which is not ideal and is due to the simple spinning device used in this study. The device had only one-stage solidification, lacked multistage washing, and did not have sufficient drafting conditions. If the equipment can be improved, the diameter of fibers might range from 30 to $40 \mu \mathrm{m}$. The pure cellulose fibers were white, whereas the fibers containing Ag NPs ranged from yellowish orange to brown as the Ag NP content increased. The color change in the fibers may be due to surface plasmon resonance in the Ag NPs in the doped fibers, indicating that more Ag NPs formed (Zhang et al. 2015; RacRumijowska et al. 2017). Additionally, as the color of the nanocomposite fibers did not change even after washing with distilled water for $48 \mathrm{~h}$, it can be inferred that stable Ag NPs were formed in situ in the fibers (Muthulakshmi et al. 2017). 

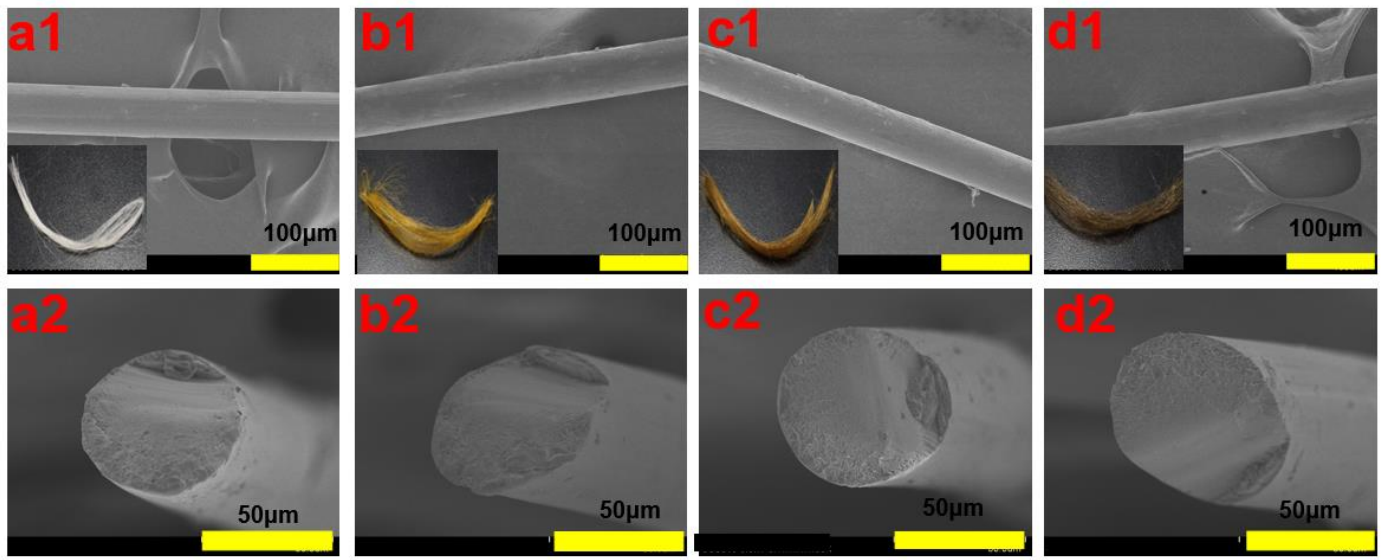

Fig. 4. SEM images (with inset photographs) of regenerated cellulose: (a1 to d1) the fiber surfaces and physical maps of R0 to R3, respectively, and (a2 to d2) the fiber cross sections of $\mathrm{R} 0$ to $\mathrm{R} 3$, respectively
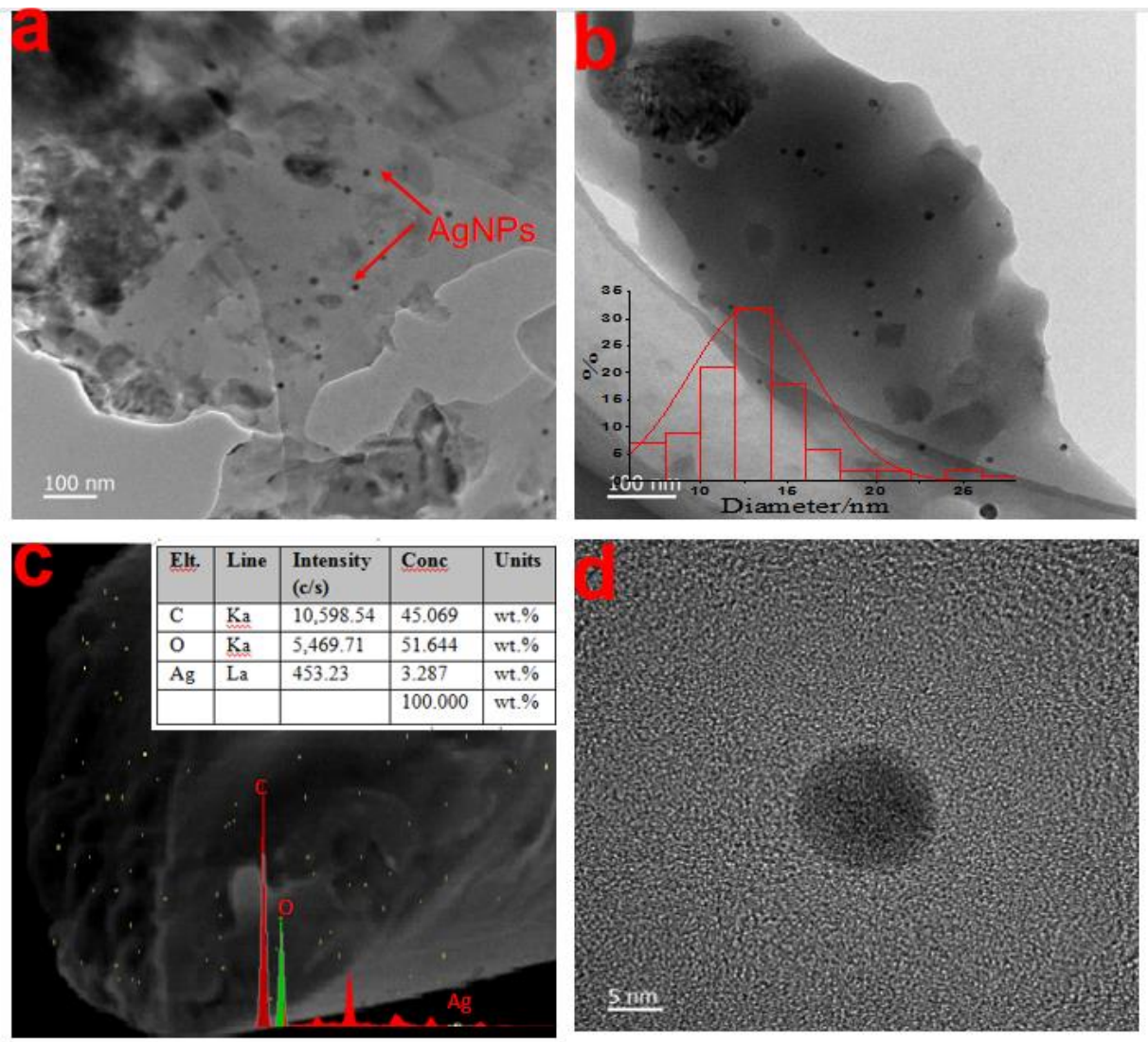

Fig. 5. (a) TEM image of the Ag NP / cellulose fibers (R3), (b) the particle size distribution of the Ag NPs (R3), (c) the dispersion of Ag NPs in the fiber cross section and EDS image (R3), and (d) high-magnification image of Ag NPs

The energy dispersive spectrum measured from SEM further confirmed that there was Ag in the composite fiber. Figure 5c contains an EDS image (Fig. S1). Additionally, the EDS image showed that the nanoparticles (yellow crystals) were uniformly distributed throughout the fiber (Fig. 5c), and the nano silver is evenly distributed on the cross section of the fiber. Transmission electron microscopy observations were performed to further 
investigate the microstructure of the Ag NPs (Fig. 5). The TEM images showed virtually spherical Ag NPs with uniform dispersion anchored to the fibers. These observations clearly indicated that Ag NPs had formed inside the matrix, and there was no aggregation of Ag NPs, which confirmed that PEI and cellulose prevented Ag NP aggregation. The size distribution of the particles was measured using Nano Measure software. The corresponding statistical histogram (Fig. 5b) showed that the sizes of the formed Ag NPs were in the approximate range of $7 \mathrm{~nm}$ to $27 \mathrm{~nm}$, with a mean diameter of $13 \mathrm{~nm}$. Most of the NPs had diameters in the range of $10 \mathrm{~nm}$ to $15 \mathrm{~nm}$. The size, morphology, and stability of Ag NPs strongly affect their antimicrobial and antiviral activity (Xia et al. 2012). Some authors have found that Ag NPs measuring $5 \mathrm{~nm}$ to $20 \mathrm{~nm}$ have greater antibacterial activities (Liu et al. 2010).

\section{Antimicrobial Effects of Cellulose / Ag NP Fibers}

Silver nanoparticle / cellulose fibers are known to have good antibacterial activity. The antibacterial activity of the prepared nanocomposite fibers was tested against the common bacteria $E$. coli and $S$. aureus using the disk diffusion method after $24 \mathrm{~h}$ of incubation at $37{ }^{\circ} \mathrm{C}$ (Fig. 6). The matrix did not show any activity; however, the cellulose I Ag NP fibers showed clear antibacterial activity compared with the matrix. For E. coli, the diameters of the clear zones were $6.5 \mathrm{~mm}, 7 \mathrm{~mm}$, and $8 \mathrm{~mm}$ for R1, R2, and R3, respectively. The diameters of the clear zones for $S$. aureus were considerably smaller than those for E. coli: $1.1 \mathrm{~mm}, 2.6 \mathrm{~mm}$, and $2.9 \mathrm{~mm}$ for R1, R2, and R3, respectively. The diameter of the inhibition zone can represent the antibacterial strength to a certain extent. The difference in the organization of the cell wall results in greater sensitivity of Gramnegative bacteria.
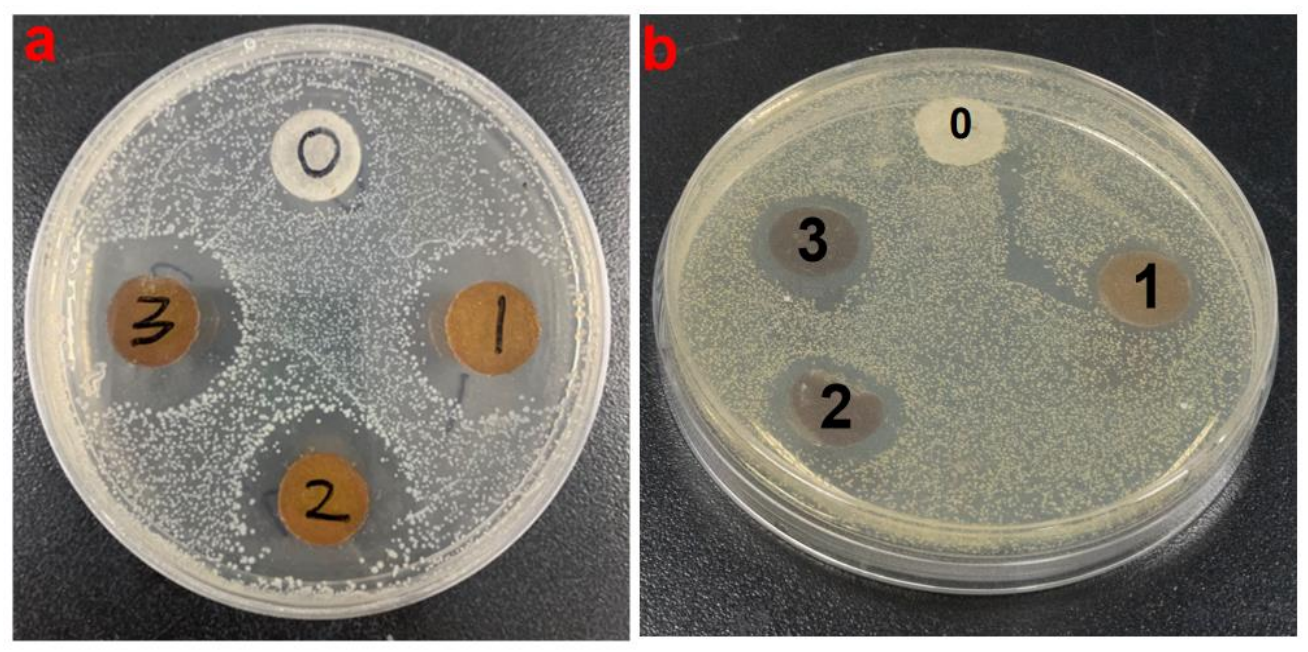

Fig. 6. Images of the antibacterial clear zone diameters for (a) E. coli and (b) S. aureus: (0 to 3$)$ samples $\mathrm{R} 0$ to $\mathrm{R} 3$ 


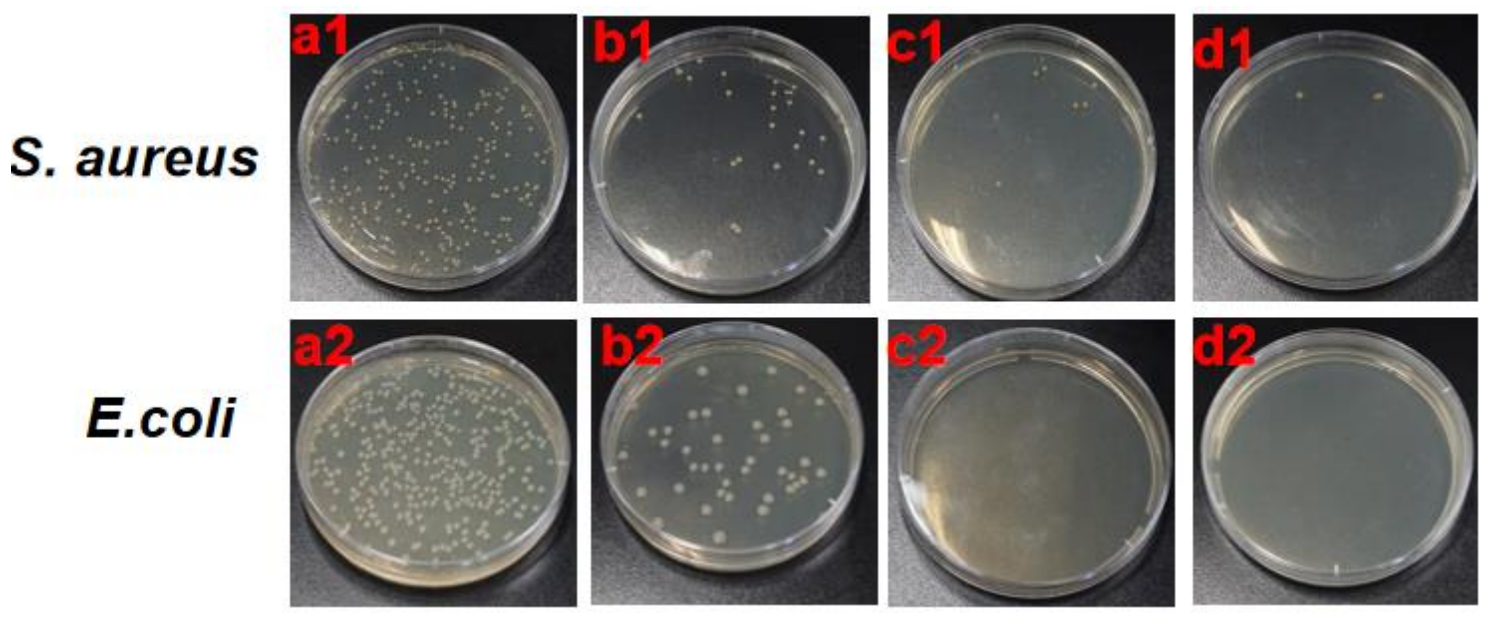

Fig. 7. Images of agar plates inoculated with (a1 to d1) S. aureus and (a2 to d2) E. coli suspensions and treated with $\mathrm{R} 0$ to $\mathrm{R} 3$, respectively

The antibacterial ratios of the Ag NP/ cellulose fibers against E. coli and S. aureus were tested using the shake flask test method. After $24 \mathrm{~h}$ of incubation, the $S$. aureus reductions for the three fibers containing different amounts of Ag NPs were $82.5 \%$, $96.25 \%$, and $98.12 \%$, for $\mathrm{R} 1, \mathrm{R} 2$, and $\mathrm{R} 3$, respectively. For E. coli, the sterilizing efficiencies of Ag NP / cellulose fibers were $89.3 \%,>99 \%$, and $>99 \%$, for R1, R2, and R3, respectively. These findings were similar to those obtained using the agar diffusion method, which indicated that the Ag NPs provide better antibacterial activity against $E$. coli. The results demonstrated that the Ag NP / cellulose fibers had good antimicrobial activities against $E$. coli (Gram negative) and $S$. aureus (Gram positive). The antibacterial mechanism of silver nanoparticles has been explained in many reports. A generally accepted view is that silver nanoparticles release silver ions and thus disrupt the physiological functions of bacteria. Silver nanoparticles have a higher specific surface area and are faster at releasing $\mathrm{Ag}^{+}$into bacteria than large silver particles, which causes more serious damage to bacteria (Shrivastava et al. 2007). The antibacterial activities against $E$. coli were higher than those against $S$. aureus, which was probably due to the difference between the cell walls of Gram-positive and Gram-negative bacteria (Rac-Rumijowska et al. 2019). It is well known that the bactericidal activity of Ag NPs is affected by the thickness and composition of the microorganism cell wall. In Gram-positive bacteria, the cell wall comprises a negatively charged peptidoglycan, and this layer is thicker than Gram-negative bacteria, which causes silver ions to remain stuck to the cell surface. Gramnegative bacteria have thinner cell walls and lower peptidoglycan contents and are therefore more sensitive to Ag NPs.

\section{CONCLUSIONS}

1. Silver nanoparticle / cellulose fibers were successfully prepared using the direct onepot synthesis of Ag NPs in the spinning solution. Tetrabutylammonium hydroxide was used not only as a solvent for cellulose but also as an accelerator for cellulose in the reduction of silver ions.

2. As the reduction time increased, Ag NPs were first generated ( $0 \mathrm{~h}$ to $24 \mathrm{~h})$ and then

Zeng et al. (2021). "Cellulose / Ag nanoparticle fibers," BioResources 16(2), 3360-3376. 3371 
agglomerated as the number of generated Ag NPs increased. Therefore, the addition of stabilizers was necessary. The formation of Ag NPs caused a slight change in the crystallinity and did not change the functional groups of cellulose.

3. The prepared regenerated cellulose fibers had high mechanical properties. Scanning electron microscopy (EDS) and transmission electron microcopy (TEM) showed that the Ag NPs, which had a mean diameter of $13 \mathrm{~mm}$, were uniformly dispersed in the R3 fibers.

4. The fibers exhibited excellent antibacterial activities against E. coli and S. aureus, as demonstrated using the inhibition zone method and shake flask test method.

5. This simple and mild synthetic method provides a new strategy for preparing highvalue-added cellulose-based nanohybrid materials.

\section{ACKNOWLEDGMENTS}

The authors are grateful for support from the Specialized Research Fund for the Forestry Public Welfare Industry (201504602-2).

\section{REFERENCES CITED}

Cao, J., Wei, W., Gou, G., Jiang, M., Cui, Y., Zhang, S., Wang, Y., and Zhou, Z. (2018). "Cellulose films from the aqueous DMSO/TBAH-system," Cellulose 25(3), 19751986. DOI: 10.1007/s10570-017-1639-5

Chaloupka, K., Malam, Y., and Seifalian, A. M. (2010). "Nanosilver as a new generation of nanoproduct in biomedical applications," Trends in Biotechnology 28(11), 580588. DOI: 10.1016/j.tibtech.2010.07.006

Chen, Q.-Y., Xiao, S.-L., Shi, S. Q., and Cai, L.-P. (2020). “A one-pot synthesis and characterization of antibacterial silver nanoparticle-cellulose film," Polymers 12(2). DOI: $10.3390 /$ polym 12020440

Chen, X., Chen, X., Cai, X.-M., Huang, S., and Wang, F. (2018). "Cellulose dissolution in a mixed solvent of tetra(n-butyl)ammonium hydroxide/dimethyl sulfoxide via radical reactions," ACS Sustainable Chemistry \& Engineering 6(3), 2898-2904. DOI: 10.1021/acssuschemeng.7b04491

Dong, Y.-Y., Fu, L.-H., Liu, S., Ma, M.-G., and Wang, B. (2015). "Silver-reinforced cellulose hybrids with enhanced antibacterial activity: Synthesis, characterization, and mechanism," RSC Advances 5(118), 97359-97366. DOI: 10.1039/c5ra19758a

Echegoyen, Y., and Nerín, C. (2013). "Nanoparticle release from nano-silver antimicrobial food containers," Food and Chemical Toxicology 62, 16-22. DOI: 10.1016/j.fct.2013.08.014

Felix, E.-M., Antoni, M., Pause, I., Schaefer, S., Kunz, U., Weidler, N., Muench, F., and Ensinger, W. (2016). "Template-based synthesis of metallic Pd nanotubes by electroless deposition and their use as catalysts in the 4-nitrophenol model reaction," Green Chemistry 18(2), 558-564. DOI: 10.1039/c5gc01356a

French, A. D. (2014). "Idealized powder diffraction patterns for cellulose polymorphs," 
Cellulose 21(2), 885-896. DOI: 10.1007/s10570-013-0030-4

Fu, F., Gu, J., Cao, J., Shen, R., Liu, H., Zhang, Y., Liu, X., and Zhou, J. (2017).

"Reduction of silver ions using an alkaline cellulose dope: Straightforward access to $\mathrm{Ag} / \mathrm{ZnO}$ decorated cellulose nanocomposite film with enhanced antibacterial activities," ACS Sustainable Chemistry \& Engineering 6(1), 738-748. DOI: 10.1021/acssuschemeng.7b03059

Johnston, J. H., and Nilsson, T. (2012). "Nanogold and nanosilver composites with lignin-containing cellulose fibres," Journal of Materials Science 47(3), 1103-1112. DOI: $10.1007 / \mathrm{s} 10853-011-5882-0$

Kannan, B., Cha, H., and Hosie, I. C. (2016). "Electrospinning-Commercial applications, challenges and opportunities," in: Nano-size Polymers: Preparation, Properties, Applications, S. Fakirov (ed.), Springer, Cham, Switzerland, pp. 309-342. DOI: 10.1007/978-3-319-39715-3-11

Kontturi, K. S., Kontturi, E., and Laine, J. (2013). "Specific water uptake of thin films from nanofibrillar cellulose," Journal of Materials Chemistry A 1(43), 13655-13663. DOI: $10.1039 / \mathrm{c} 3$ ta12998e

Lee, H. J., Lee, S. G., Oh, E. J., Chung, H. Y., Han, S. I., Kim, E. J., Seo, S. Y., Ghim, H. D., Yeum, J. H., and Choi, J. H. (2011). "Antimicrobial polyethyleneimine-silver nanoparticles in a stable colloidal dispersion," Colloids and Surfaces B: Biointerfaces 88(1), 505-511. DOI: 10.1016/j.colsurfb.2011.07.041

Li, S., and Huang, J. (2016). "Cellulose-rich nanofiber-based functional nanoarchitectures," Advanced Materials 28(6), 1143-1158. DOI: 10.1002/adma.201501878

Liang, M., Su, R., Huang, R., Qi, W., Yu, Y., Wang, L., and He, Z. (2014). "Facile in situ synthesis of silver nanoparticles on procyanidin-grafted eggshell membrane and their catalytic properties," ACS Applied Materials \& Interfaces 6(7), 4638-4649. DOI: 10.1021/am500665p

Liu, W., Wu, Y., Wang, C., Li, H. C., Wang, T., Liao, C. Y., Cui, L., Zhou, Q. F., Yan, B., and Jiang, G. B. (2010). "Impact of silver nanoparticles on human cells: Effect of particle size," Nanotoxicology 4(3), 319-330. DOI: 10.3109/17435390.2010.483745

Liu, Z., Wang, H., Li, Z., Lu, X., Zhang, X., Zhang, S., and Zhou, K. (2011).

"Characterization of the regenerated cellulose films in ionic liquids and rheological properties of the solutions," Materials Chemistry and Physics 128(1-2), 220-227.

DOI: 10.1016/j.matchemphys.2011.02.062

Medronho, B., Filipe, A., Napso, S., Khalfin, R. L., Pereira, R. F. P., de Zea Bermudez, V., Romano, A., and Cohen, Y. (2019). "Silk fibroin dissolution in tetrabutylammonium hydroxide aqueous solution," Biomacromolecules 20(11), 41074116. DOI: $10.1021 /$ acs.biomac.9b00946

Miao, J., Yu, Y., Jiang, Z., and Zhang, L. (2016). "One-pot preparation of hydrophobic cellulose nanocrystals in an ionic liquid," Cellulose 23(2), 1209-1219. DOI: 10.1007/s10570-016-0864-7

Muthulakshmi, L., Rajini, N., Varada Rajalu, A., Siengchin, S., and Kathiresan, T. (2017). "Synthesis and characterization of cellulose/silver nanocomposites from bioflocculant reducing agent," International Journal of Biological Macromolecules 103, 11131120. DOI: 10.1016/j.ijbiomac.2017.05.068

Nangmenyi, G., Yue, Z., Mehrabi, S., Mintz, E., and Economy, J. (2009). "Synthesis and characterization of silver-nanoparticle-impregnated fiberglass and utility in water disinfection," Nanotechnology 20(49). DOI: 10.1088/0957-4484/20/49/495705 
Nishimura, S., Mott, D., Takagaki, A., Maenosono, S., and Ebitani, K. (2011). "Role of base in the formation of silver nanoparticles synthesized using sodium acrylate as a dual reducing and encapsulating agent," Physical Chemistry Chemical Physics 13(20), 9335-9343. DOI: 10.1039/c0cp02985h

Rac-Rumijowska, O., Fiedot, M., Karbownik, I., Suchorska-Woźniak, P., and Teterycz, H. (2017). "Synthesis of silver nanoparticles in NMMO and their in situ doping into cellulose fibers," Cellulose 24(3), 1355-1370. DOI: 10.1007/s10570-016-1168-7

Rac-Rumijowska, O., Maliszewska, I., Fiedot-Toboła, M., Karbownik, I., and Teterycz, H. (2019). "Multifunctional nanocomposite cellulose fibers doped in situ with silver nanoparticles," Polymers 11(3). DOI: 10.3390/polym11030562

Rogina-Car, B., Budimir, A., Turcic, V., and Katovic, D. (2014). "Do multi-use cellulosic textiles provide safe protection against the contamination of sterilized items?," Cellulose 21(3), 2101-2109. DOI: 10.1007/s10570-014-0199-1

Seo, E., Kim, J., Hong, Y., Kim, Y. S., Lee, D., and Kim, B.-S. (2013). “Double hydrophilic block copolymer templated Au nanoparticles with enhanced catalytic activity toward nitroarene reduction," The Journal of Physical Chemistry C 117(22), 11686-11693. DOI: 10.1021/jp4027139

Shrivastava, S., Bera, T., Roy, A., Singh, G., Ramachandrarao, P., and Dash, D. (2007). "Characterization of enhanced antibacterial effects of novel silver nanoparticles," Nanotechnology 18(22). DOI: 10.1088/0957-4484/18/22/225103

Song, J. Y., and Kim, B. S. (2009). "Rapid biological synthesis of silver nanoparticles using plant leaf extracts," Bioprocess and Biosystems Engineering 32(1), 79-84. DOI: 10.1007/s00449-008-0224-6

Taurozzi, J. S., Arul, H., Bosak, V. Z., Burban, A. F., Voice, T. C., Bruening, M. L., and Tarabara, V. V. (2008). "Effect of filler incorporation route on the properties of polysulfone-silver nanocomposite membranes of different porosities," Journal of Membrane Science 325(1), 58-68. DOI: 10.1016/j.memsci.2008.07.010

Tran, C. D., Prosenc, F., Franko, M., and Benzi, G. (2016). "One-pot synthesis of biocompatible silver nanoparticle composites from cellulose and keratin: Characterization and antimicrobial activity," ACS Applied Materials \& Interfaces 8(50), 34791-34801. DOI: 10.1021/acsami.6b14347

Tsai, T.-T., Huang, T.-H., Chang, C.-J., Ho, N. Y.-J., Tseng, Y.-T., and Chen, C.-F. (2017). "Antibacterial cellulose paper made with silver-coated gold nanoparticles," Scientific Reports 7(1). DOI: 10.1038/s41598-017-03357-w

Wu, J., Zheng, Y., Song, W., Luan, J., Wen, X., Wu, Z., Chen, X., Wang, Q., and Guo, S. (2014). "In situ synthesis of silver-nanoparticles/bacterial cellulose composites for slow-released antimicrobial wound dressing," Carbohydrate Polymers 102, 762-771. DOI: 10.1016/j.carbpol.2013.10.093

Xia, X., Zeng, J., Zhang, Q., Moran, C. H., and Xia, Y. (2012). "Recent developments in shape-controlled synthesis of silver nanocrystals," The Journal of Physical Chemistry C 116(41), 21647-21656. DOI: 10.1021/jp306063p

Yang, S., Wang, T., Tang, R., Yan, Q., Tian, W., and Zhang, L. (2020). "Enhanced permeability, mechanical and antibacterial properties of cellulose acetate ultrafiltration membranes incorporated with lignocellulose nanofibrils," International Journal of Biological Macromolecules 151, 159-167. DOI: 10.1016/j.ijbiomac.2020.02.124

Yuan, Q., Li, N., Geng, W., Chi, Y., and Li, X. (2012). "Preparation of magnetically recoverable $\mathrm{Fe}_{3} \mathrm{O}_{4} @ \mathrm{SiO}_{2} @$ meso- $\mathrm{TiO}_{2}$ nanocomposites with enhanced photocatalytic

Zeng et al. (2021). "Cellulose / Ag nanoparticle fibers," BioResources 16(2), 3360-3376. 3374 
ability," Materials Research Bulletin 47(9), 2396-2402. DOI:

10.1016/j.materresbull.2012.05.031

Zhang, H., Duan, T., Zhu, W., and Yao, W.-T. (2015). "Natural chrysotile-based nanowires decorated with monodispersed Ag nanoparticles as a highly active and reusable hydrogenation catalyst," The Journal of Physical Chemistry C 119(37), 21465-21472. DOI: 10.1021/acs.jpcc.5b05450

Zhong, C., Cheng, F., Zhu, Y., Gao, Z., Jia, H., and Wei, P. (2017). "Dissolution mechanism of cellulose in quaternary ammonium hydroxide: Revisiting through molecular interactions," Carbohydrate Polymers 174, 400-408. DOI: 10.1016/j.carbpol.2017.06.078

Article submitted: January 4, 2021; Peer review completed: February 13, 2021; Revised version received and accepted: February 24, 2021; Published: March 22, 2021.

DOI: 10.15376/biores.16.2.3360-3376 


\section{APPENDIX: SUPPLEMENTARY MATERIAL}

One-pot Synthesis of Cellulose / Silver Nanoparticles Fibers and Antibacterial Application

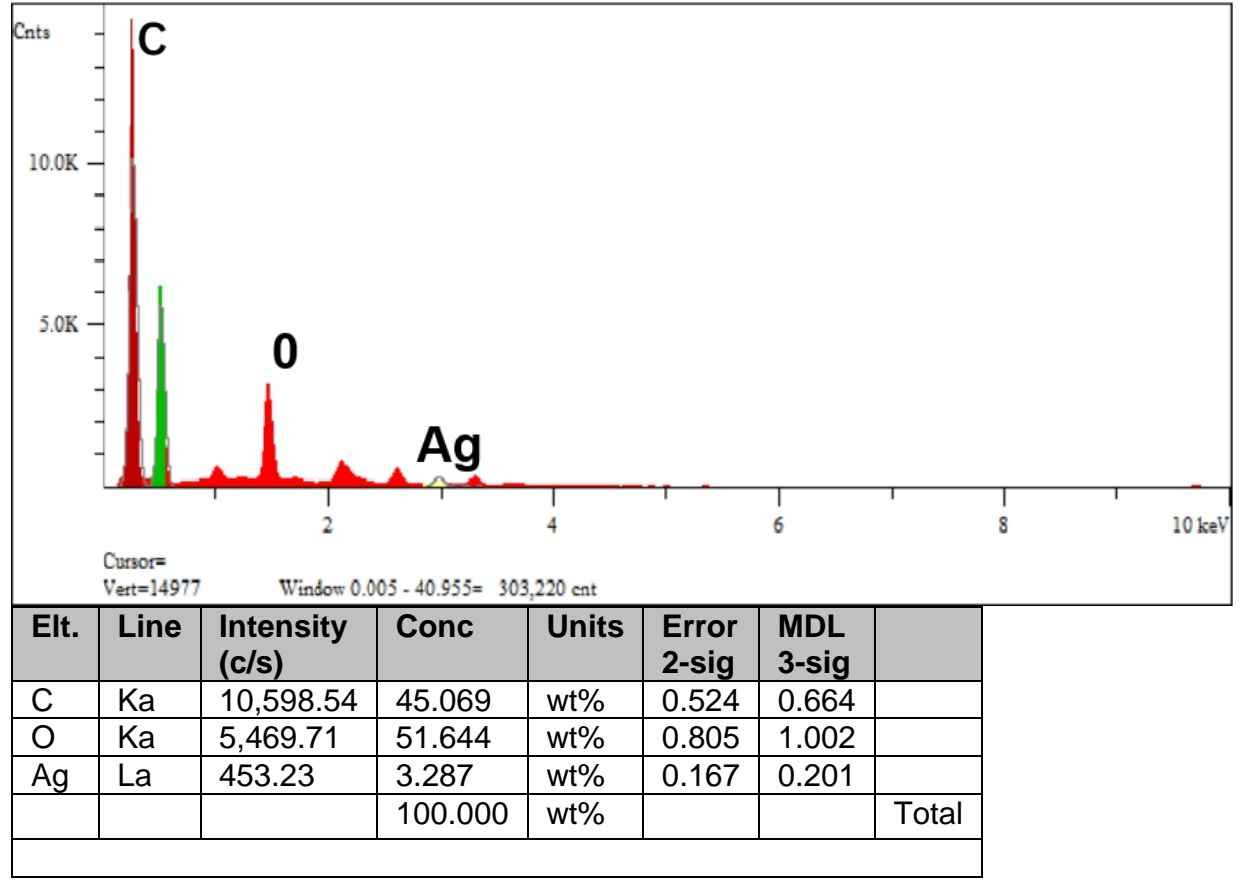

SEM

C

0

$\mathrm{Ag}$

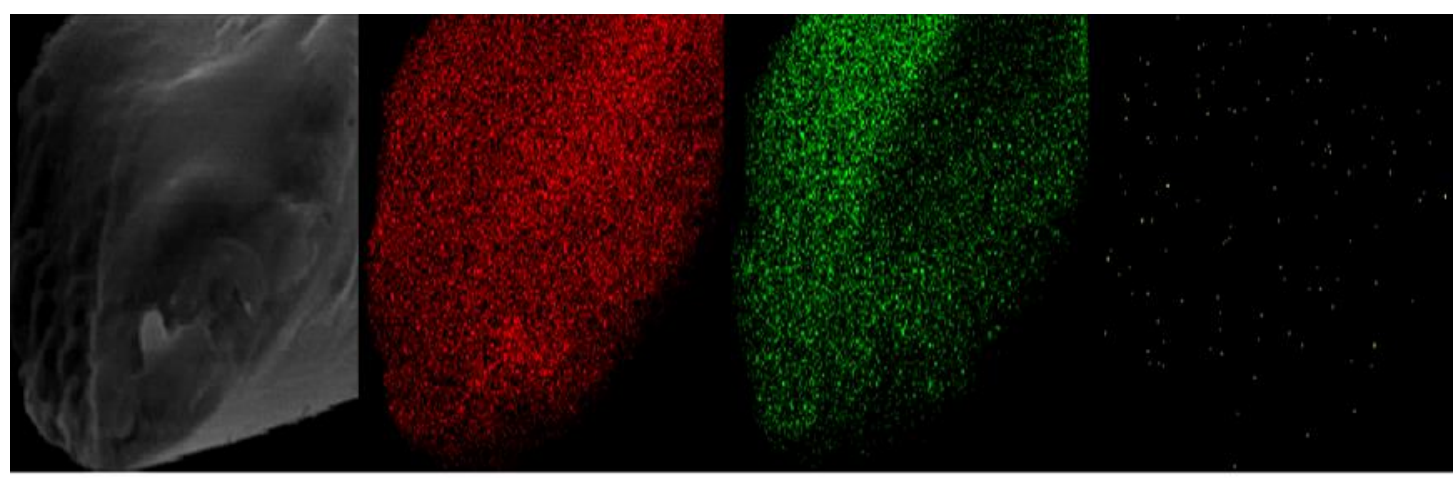

Fig. S1. EDS images of section of R3 fibers 